\title{
An Improved Fellegi-Sunter Framework for Probabilistic Record Linkage Between Large Data Sets
}

\author{
Marco Fortini ${ }^{1}$
}

\begin{abstract}
Record linkage addresses the problem of identifying pairs of records coming from different sources and referred to the same unit of interest. Fellegi and Sunter propose an optimal statistical test in order to assign the match status to the candidate pairs, in which the needed parameters are obtained through EM algorithm directly applied to the set of candidate pairs, without recourse to training data. However, this procedure has a quadratic complexity as the two lists to be matched grow. In addition, a large bias of EM-estimated parameters is also produced in this case, so that the problem is tackled by reducing the set of candidate pairs through filtering methods such as blocking. Unfortunately, the probability that excluded pairs would be actually true-matches cannot be assessed through such methods.

The present work proposes an efficient approach in which the comparison of records between lists are minimised while the EM estimates are modified by modelling tables with structural zeros in order to obtain unbiased estimates of the parameters. Improvement achieved by the suggested method is shown by means of simulations and an application based on real data.
\end{abstract}

Key words: Structural zeros; robustness; EM algorithm; blocking.

\section{Introduction}

Record linkage (RL) consists of identifying pairs of records concerning the same individual, henceforth called matches, when these are included in different files or are duplicates in the same file. Matching is established by comparing a group of variables (key variables) that are common to the records and combined into a unique identifier. When the identifier is perfectly accurate the problem can be solved quite easily, and gains statistical interest if the key variables are affected by measurement errors.

Newcombe et al. (1959) laid the foundations for the probabilistic setting of the record linkage, while Fellegi and Sunter (1969) defined an optimal test for the identification of the matches amongst all the pairs of the Cartesian product between the files to be linked. The Fellegi-Sunter theory remains at the core of most of the current applications. In their approach, the statistical parameters of the test are estimated by the method of moments applied to the frequency distribution of the pairs from the Cartesian product by pattern of agreement between key variables. In so doing, the estimates are obtained without referring to a training set in which the match status of the pairs is known in advance. Winkler (1988) and Jaro (1989) improved the estimation procedure by introducing latent class models

\footnotetext{
${ }^{1}$ Italian National Institute of Statistics (Istat) - Directorate for Methodology and Statistical Process Design, Via C. Balbo, 16, 00184, Rome, Italy. Email: fortini@istat.it
} 
(LCM), in which dichotomous indicators of agreement amongst key variables are used to identify the two-class latent variable that constitute the matching status of the pairs through the Expectation-Maximisation (EM) algorithm (Dempster et al. 1977).

As the size of the files to be linked grow, the size of their Cartesian product increases in a quadratic way, while the number of matches remains roughly proportional to the smaller of the two files. In these circumstances, the parameters of the LCM become biased quite early due to the disproportion between matches and non-matches and, thus, become useless to the purpose of record linkage.

This drawback in the estimation process is found well before the size of the pairs to be examined reaches the limits of the computational power available nowadays. As a matter of fact, it already occurs when the proportion $p$ of matches out of the pairs of the Cartesian product falls below the limit of 5\% (Winkler 2006) and key variables are not highly informative.

Empirical studies with the record linkage toolkit RELAIS (Cibella et al. 2009) developed by Italian National Institute of Statistics (Istat) show that using highly discriminant key variables, the above mentioned proportion $p$ can be reduced up to $0.5-0.1 \%$ before it leads to the misclassification of matched and non-matched pairs and to the instability of the estimates. In other words, if we consider two files of the same size, a proportion $\mathrm{p}$ of $5 \%$ would limit file sizes to only $0.05^{-1}=20$ units (two files of 20 records that produce a Cartesian product of 400 pairs), that could increase until 1,000 records (with a Cartesian product of a million pairs) in the best-case scenario, where estimates remain unbiased until a match rate of $0.1 \%$. In order to go beyond this limit, Yancey (2002) proposed to enrich the density of matches in the set of checked pairs to obtain improved EM algorithm estimates for the record linkage conditional probability parameters.

In real applications, files of hundreds of thousands or even millions of records often have to be linked together, resulting in Cartesian product of thousands of billions of pairs and making the problem complicated quite early also in terms of computational resources. For these reasons, filtering (or indexing) methods have been largely proposed in literature to discard large numbers of the pairs with low evidence of being matches (Christen 2012 chap. 4) before the linkage estimation phase. Besides the great operational value achieved by these procedures (Baxter et al. 2003), filtering methods represent a step backwards from the probabilistic approach, since they condition the linkage probabilities to a subset of Cartesian product chosen with a deterministic criterion (Murray 2015). The adoption of this approach does not enable us to assess the risk of erroneous exclusion of real matches from the probabilistic linkage. In addition, it could cause false matches for those records whose true match pair was excluded from the analysis because of previous faults in the filtering process. Murray (2015) adapted estimation procedures for linkage parameter so as to take into account conditioning due to filtering process.

The contributions of this work are two-fold:

1. First of all, a sample filtering criterion of a subset of pairs that is representative of the entire Cartesian product of the two files to be combined is proposed. Unlike traditional filtering methods, a sampling approach makes it possible to evaluate matching probabilities for all of the pairs of the Cartesian product; 
2. Secondly, the LCM parameters estimation are adapted through a robust EM approach which improves the standard one, as it remains unbiased when the proportion $p$ of matches over the size of the Cartesian set becomes very small.

In this way, the number of pairs examined is reduced while the parameter estimation remains representative of the entire set of possible pairs. Although the robust estimation method and the sample-based filtering criterion are presented jointly in this article, it is worth noting that the former is also valid without the latter and can be used either with any other filtering criteria or along with the whole Cartesian set.

The remainder of the present article is organised as follows: Section 2 introduces the sample-based filtering approach after a formal definition of the record linkage and a description of the contingency table of pairs by their patterns of agreement between key variables, which is required for estimation purposes. Subsequently, in Section 3, the standard estimation method for record linkage parameters and its robust counterpart are shown. In Section 4, the accuracy of the present proposal is investigated by means of a simulation study, while Section 5 describes a real data example in which the method is implemented and is compared with a standard estimation approach based on traditional filtering. Lastly, in the final Section 6, some advantages and drawbacks are discussed, as well as potential further research to be carried out.

\section{The Record Linkage Setting and the Dataset Under the Sample-Based Filtering Approach}

In the first part of this section the canonical probabilistic record linkage is introduced along with a description of the data structure needed for the parameter estimation which will be described in Section 3.

As mentioned in the introduction, a simplistic matching of two files requires comparing each pair of records, which is inefficient and infeasible, particularly when files are large. As such, it is important to use some techniques to reduce the number of comparisons required. For our purposes, we use filtering, which first cycles through the record pairs and retains only those that match on at least a certain number of key variables. Filtering, then, assigns zero probability to records that do not match as they are considered extremely rare and discard them from the subsequent analyses. However, filtering does not allow to verify how actually close to zero is the probability of the excluded matches. Therefore, in the remainder of Section 2 a sample-based filtering approach is described as a way to make inferences on the complete Cartesian product when its size explodes due to the growth of the files to be merged.

Fellegi and Sunter (1969) consider record linkage as a discriminant analysis that, following their notation, aims to determine if a given pair of records $(a, b)$, coming from the Cartesian product $\Omega$ between two files A and B to be linked, refers to the same unit. Let us denote as $\gamma=\left\{\gamma_{1}, \ldots, \gamma_{k}\right\}$ the $\mathrm{K}$ values vector (or pattern) of agreement $\left(\gamma_{i}=1\right)$ or disagreement $\left(\gamma_{i}=0\right)$ between $\mathrm{K}$ couples of common key variables $\left(\mathrm{X}_{\mathrm{i}, a}, \mathrm{X}_{\mathrm{i}, b} ; \mathrm{i}=\right.$ $1, \ldots, \mathrm{K},(a, b) \in \Omega)$ measured on each pair of records $(a, b) \in \Omega)$. We also denote $\Gamma$ the set of $2^{K}$ patterns $\gamma$ given by all the possible combinations of agreement/disagreement between the K key variables. Moreover, let us define as "matched" those pairs $(a, b)$ whose records refer to the same unit and call $M$ the corresponding set of matched pairs. Similarly, 
we define the set $U$ as consisting of the pairs linking two different units and called "unmatched" pairs, with $\Omega=M \cup U$, and $M \cap U=\varnothing$.

The probabilities that the pair $(a, b) \in \Omega$ ) has a given pattern of agreement $\gamma$ given its match status are indicated as $m_{\gamma}=\mathrm{P}(\gamma \mid(a, b) \in M)$ for matched pair $(a, b) \in M$ and $u_{\gamma}=\mathrm{P}(\gamma \mid(a, b) \in U)$ for unmatched pair $(a, b) \in U$, while $p=P((a, b) \in M)$ represents the marginal proportion of matches $(a, b) \in M$ out of $\Omega$.

Given the above notation, the likelihood ratio test $\mathrm{r}_{\gamma}=\frac{m_{\gamma}}{u_{\gamma}}$ is used by Fellegi and Sunter to assign the unknown matching status to pairs showing the vector $\gamma$. A pair is tagged as 'matched' when its corresponding $\mathrm{r}_{\gamma}$ is larger than a fixed threshold $\lambda_{M}$, and labelled instead as 'unmatched' when its rate $\mathrm{r}_{\gamma}$ is smaller than another fixed threshold $\lambda_{U} \leq \lambda_{M}$. When $\lambda_{M}>\lambda_{U}$ a certain number of pairs whose corresponding $\mathrm{r}_{\gamma}$ do not comply with none of the two limits, the pairs remain undecided and are sent to clerical review to be resolved. They showed that $\mathrm{r}_{\gamma}$ is the best among all the possible test statistics for the same problem and define a criterion to set $\lambda_{M}$ and $\lambda_{U}$ based on expected false positive and false negative error probabilities caused by wrong decisions, so as to obtain the right trade-off between the risk of error and the clerical review efforts.

It is important to note that pairs having the same pattern of agreement share the evidence of being matches, thus, all available information on the pairs' status is enclosed in the frequency distribution of the pairs $(a, b) \in \Omega$ according to their $\gamma$ pattern. In Section 3, we will see how probabilities $m_{\gamma}, u_{\gamma}, \forall \gamma$, and $p$ - which were needed to accomplish the test can be estimated from the frequency distribution of the pairs in $\Omega$ according to their patterns of agreement $\gamma$. Here we describe the characteristics of the frequency distribution of pairs, showed without loss of generality in Table 1 for three key variables, where 1 stands for agreement and 0 for disagreement. Subsequently, we will see how the table can be inferred under the sample-based filtering approach.

The first three columns of Table 1 describe the comparison vectors $\gamma$, with $\gamma_{i} \in(0,1), \quad i=1,2,3$. The fourth column represents the absolute frequencies of pairs $N_{\gamma}$ within the pattern $\gamma$, with $\Sigma_{\gamma} N_{\gamma}=N_{\Omega}$, while the last column reports the relative frequencies. When K key variables are available, the frequency distribution is made up by $2^{K}$ frequency cells corresponding to all the possible combinations of agreement/ disagreement patterns. When $\Omega$ increases due to the increasing size of the files, a sample-

Table 1. Contingency table of pairs by three key variables

\begin{tabular}{lllll}
\hline$\gamma_{1}$ & $\gamma_{2}$ & $\gamma_{3}$ & $N_{\gamma}$ & $p_{\gamma}$ \\
\hline 1 & 1 & 1 & $N_{111}$ & $p_{111}$ \\
1 & 1 & 0 & $N_{110}$ & $p_{110}$ \\
1 & 0 & 1 & $N_{101}$ & $p_{101}$ \\
0 & 1 & 1 & $N_{011}$ & $p_{011}$ \\
0 & 0 & 1 & $N_{001}$ & $p_{001}$ \\
0 & 1 & 0 & $N_{010}$ & $p_{010}$ \\
1 & 0 & 0 & $N_{100}$ & $p_{100}$ \\
0 & 0 & 0 & $N_{000}$ & $p_{000}$ \\
\hline & Tot & & $N_{\Omega}$ & 1 \\
\hline
\end{tabular}


based filtering of pairs from $\Omega$ is proposed in order to estimate the frequency distribution by the patterns $\gamma$, instead of investigating the whole set of pairs.

It is worth mentioning that in real cases, the comparison patterns separate matched and unmatched pairs quite well to the extent that unmatched pairs are expected to be found mainly within patterns with high disagreement. Similarly, matched pairs will fall mainly in patterns with little or no discrepancies. Since the set of unmatched pairs $U$ is widely larger than the set $\mathrm{M}$ of matched pairs, the frequency distribution will concentrate toward the most discordant patterns. For this reason, a random sample would fail to accurately estimate the frequency cells for patterns showing large agreement. Consequently, our sampling approach is combined with the complete enumeration of pairs whose disagreement does not exceed one key variable. Full enumeration is obtained through $\mathrm{K}$ repeated merge tasks between the two files to be matched. This can be considered as a multiple blocking that uses all the sets of K-1 key variables adopted for parameters estimation on a rotating basis. Unlike standard multiple blocking, which recurs to variables (or coarser classifications) other than those used as keys at linkage stage, it is possible to do so because sampling from Cartesian product allows the shaping of comparisons table and parameters estimation, which would be impossible for conventional blocking.

Quite similarly to multiple blocking and other deterministic filtering techniques, this procedure allows for great computational savings in comparison to the processing of the whole set of pairs. Moreover, our approach outperforms the traditional filtering techniques as it allows for the evaluation of matching probabilities $m_{\gamma}, u_{\gamma}, \forall \gamma$ and $p$ representative of the entire $\Omega$ through a robust EM approach, as will be explained in the next section.

The result of this procedure returns the quantities showed in Table 2 in the case of three comparison variables and is formally defined in the remainder of the present section for a generic number $\mathrm{K}$ of key variables.

More formally, in the sample-based filtering approach the frequencies $N_{\gamma}$ of pairs $(a, b) \in \Omega$ by their pattern $\gamma$ are entirely computed (or "solved" in what follows) only on the $\mathrm{K}+1$ the patterns $\gamma \in \Gamma^{S}$, where at least all the $\mathrm{K}$ key variables but one agree; for this reason, $\Gamma^{S}$ will be mentioned in the following as the set of solved patterns. Conversely, for residual patterns $\gamma \in \Gamma^{R}$, where the pairs disagree for more than one key variable, just the

Table 2. Estimated contingency table of the pairs by three key variables

\begin{tabular}{lllll}
\hline$\gamma_{1}$ & $\gamma_{2}$ & $\gamma_{3}$ & $N_{\gamma}$ & $p_{\gamma}$ \\
\hline 1 & 1 & 1 & $N_{111}$ & $p_{111}$ \\
1 & 1 & 0 & $N_{110}$ & $p_{110}$ \\
1 & 0 & 1 & $N_{101}$ & $p_{101}$ \\
0 & 1 & 1 & $N_{011}$ & $p_{011}$ \\
0 & 0 & 1 & $\hat{N}_{001}$ & $\hat{p}_{001}$ \\
0 & 1 & 0 & $\hat{N}_{010}$ & $\hat{p}_{010}$ \\
1 & 0 & 0 & $\hat{N}_{100}$ & $\hat{p}_{100}$ \\
0 & 0 & 0 & $\hat{N}_{000}$ & $\hat{p}_{000}$ \\
\hline & Tot & & $N_{\Omega}$ & 1 \\
\hline
\end{tabular}


overall number of pairs $N_{\Gamma^{R}}=\cup_{\gamma \in \Gamma^{R}} N_{\gamma}$ can be determined from

$$
N_{\Omega}=\sum_{\gamma \in \Gamma^{S}} N_{\gamma}+N_{\Gamma^{R}}
$$

where $\Gamma^{S} \cup \Gamma^{R}=\Gamma, \Gamma^{S} \cup \Gamma^{R}=\varnothing$.

The frequencies $N_{\gamma}, \forall \gamma \in \Gamma^{S}$, can be operationally achieved through the following procedure:

1. execute $\mathrm{K}$ merge routines between the two files, one for each pattern $\gamma$ that shows agreement on exactly K-1 variables, and find out the $\mathrm{K}$ amounts $M_{\gamma}, \forall \gamma \in \Gamma^{S}$, representing the number of pairs that are matched on each merge (many-to-many links are admitted),

2. calculate the number of pairs $N_{\gamma^{+}}$falling in the intersection of the merges made during the previous step 1 , with $N_{\gamma^{+}}$representing the frequency for the pattern $\gamma^{+}=\left\{\gamma_{k}=1, \forall k \in(1, K)\right\}$ of agreement on all the $\mathrm{K}$ variables,

3. assign $N_{\gamma}=M_{\gamma}-N_{\gamma^{+}}$, for all that $\gamma$ showing a pattern of agreement of order K-1,

4. being $N_{\Omega}=N_{A} N_{B}$ the product of records $N_{A}, N_{B}$ in the two dataset to be linked, the residual frequency $N_{\Gamma^{R}}$ is finally obtained from the formula (1).

Starting from a computational effort of $O\left(N^{2}\right)$ needed for comprehensive recognition of the pairs in $\Omega$, in this way computations are reduced to the order of $\mathrm{K}$ times merge/sort routines, as the computational complexity of a merge/sort algorithm is $O(N \cdot \log (N))$ (Cormen et al. 2009).

In addition, a simple random sample $\Omega *$ of $n$ pairs is selected from $\Omega$, with distribution $n_{\gamma}$ for $\gamma \in \Gamma$. The sample is then combined with the frequencies $N_{\gamma}\left(\forall \gamma \in \Gamma^{S}\right)$ obtained from points 1-4 above to estimate the relative frequencies $p_{\gamma}\left(\forall \gamma \in \Gamma^{R}\right)$ via the proportion

$$
\hat{p}_{\gamma}=\frac{\left(N_{\Omega}-N_{\Gamma^{S}}\right)}{N_{\Omega}} \cdot \frac{n_{\gamma}}{\sum_{\gamma \in \Gamma^{R}} n_{\gamma}}, \quad \forall \gamma \in \Gamma^{R} .
$$

The estimation of the frequencies $N_{\gamma}, \forall \gamma \in \Gamma^{R}$, is obtained through $\hat{N}_{\gamma}=N_{\Omega} \hat{p}_{\gamma}$, so as to shape data as shown in Table 2 for 3 key variables without loss of generality.

\section{The Robust Parameter Estimation Method}

In this section a proposal for robust estimation is presented after a brief illustration of the standard procedure for estimating the probability of record linkage.

In the traditional setting the conditional probabilities $m_{\gamma}=P(\gamma \mid(a, b) \in M)$, $u_{\gamma}=P(\gamma \mid(a, b) \in U)$, as well as the marginal proportion of matches in $\Omega$, $p=((a, b) \in M)$, are estimated (Jaro 1989) through the EM algorithm on the basis of the observed data approach and conditional independence assumptions

$$
m_{\gamma}=\prod_{k} m_{k} ; \quad u_{\gamma}=\prod_{k} u_{k} ; \quad \forall \gamma
$$


where

$$
m_{k}=\operatorname{Pr}\left(\gamma_{k}=1 \mid(a, b) \in M\right), \quad u_{k}=\operatorname{Pr}\left(\gamma_{k}=1 \mid(a, b) \in U\right), \quad k=1, \ldots, K .
$$

It is important to note, here and hereafter, the difference between the marginal probabilities $m_{k}$ and $u_{k}$, for $k=1, \ldots, K$, referred to single key variables and the combined probabilities $m_{\gamma}$ and $u_{\gamma}, \forall \gamma$, concerning patterns.

To such purpose, Jaro define the 'augmented' likelihood for the complete dataset in which the proportion of matches $g_{\gamma}$ among $N_{\gamma}$ is known for every $\gamma$

$$
l\left(g_{\gamma}, N_{\gamma} \mid \boldsymbol{m}, \boldsymbol{u}, p\right)=p^{N_{\mathrm{M}}}\left(\prod_{\gamma} m_{\gamma}^{g_{\gamma} N_{\gamma}}\right)(1-p)^{\left(N_{\Omega}-N_{M}\right)}\left(\prod_{\gamma} u_{\gamma}^{\left(1-g_{\gamma}\right) N_{\gamma}}\right) .
$$

Since, as a matter of fact, the $g_{\gamma}$ proportions are unknown, the maximum of the likelihood in (3) cannot be directly estimated and the estimates are achieved through an EM approach. After the $2 \cdot K+1$ starting values $m_{k}^{0}, u_{k}^{0}$, for $k=1, \ldots, K$, and $p^{0}$ are assigned, the step $\mathrm{E}$ is carried out to compute the expected values

$$
g_{\gamma}^{0}=P^{0}((a, b) \in M \mid \gamma)=\frac{m_{\gamma}^{0} p^{0}}{m_{\gamma}^{0} p^{0}+u_{\gamma}^{0}\left(1-p^{0}\right)}, \quad \forall \gamma
$$

under the conditional independence assumption (2).

Once the expected values are obtained for $g_{\gamma}$, they can be used to maximise the likelihood in (3) during the step $\mathrm{M}$ so as to update the parameters for $k=1, \ldots, K$. It can be shown (Jaro 1989) that MLE parameters are given by

$$
p^{1}=\frac{\sum_{\gamma} g_{\gamma}^{0} N_{\gamma}}{N_{\Omega}}, \quad m_{k}^{1}=\frac{\sum_{\gamma \cdot \gamma_{k}=1} g_{\gamma}^{0} N_{\gamma}}{p^{0} N_{\Omega}}, \quad u_{k}^{1}=\frac{\sum_{\gamma: \gamma_{k}=1}\left(1-g_{\gamma}^{0}\right) N_{\gamma}}{\left(1-p^{0}\right) N_{\Omega}}
$$

when conditional independence (2) is assumed. The procedure, which is iterated until convergence is achieved, is proven to be stable and fairly insensitive to the starting values under conditional independence assumptions (Jaro 1989; Winkler 1988).

The robust procedure, described in what follows, deals with the bias affecting the standard method when $\Omega$ becomes larger and the pairs $(a, b) \in M$ become very small in comparison with those in the whole $\Omega$. The robust EM procedure uses multinomial models with incomplete tables (Bishop et al. 1975) for estimation of $m_{k}$ and $u_{k}$ parameters, $k=1, \ldots, \ldots$, during the $\mathbf{M}$ step of the EM. Step $\mathbf{M}$ is hereinafter mentioned in bold in order to be distinguished from the set $M$ of the matched pairs.

As usual, the procedure begins with starting values $m_{k}^{0}$ and $u_{k}^{0}, k=1, \ldots, K$, and $p^{0}$, so that the E step is carried out by computing the expected values

$$
g_{\gamma}^{0}=P^{0}((a, b) \in M \mid \gamma)=\frac{m_{\gamma}^{0} p^{0}}{m_{\gamma}^{0} p^{0}+u_{\gamma}^{0}\left(1-p^{0}\right)}, \quad \forall \gamma \in \Gamma .
$$


Subsequently, the $\mathbf{M}$ step updates the probability $p$ by using $N_{\gamma}, \gamma \in \Gamma^{S}$, by means of solved patterns along with estimated frequencies $\hat{N}_{\gamma}$ for $\gamma \in \Gamma^{R}$ from residual patterns

$$
p^{1}=\frac{\sum_{\gamma \in \Gamma^{S}}\left(g_{\gamma}^{0} N_{\gamma}\right)+\sum_{\gamma \in \Gamma^{R}}\left(g_{\gamma}^{0} \hat{N}_{\gamma}\right)}{N_{\Omega}} .
$$

Now the $\mathbf{M}$ step for $m_{k}$ and $u_{k}$ estimation is modified to prevent the bias due to the disproportion between the sets $M$ and $U$. It should be observed that, given the expected proportions $g_{\gamma}^{0}$ already determined at step E, the parameters $m_{k}$ and $u_{k}$ can always be estimated independently of each other by their respective part of the likelihood function in (3). For this reason, a description of how to obtain the $m_{k}$ estimates will be provided in what follows.

Instead of using all the frequencies as in the standard case, robust approach estimates the $m_{k}, k=1, \ldots, K$, only from the $\mathrm{K}+1$ expected counts $g_{\gamma}^{0} N_{\gamma}$ for $\gamma \in \Gamma^{S}$, from solved patterns while the remaining patterns $\gamma \in \Gamma^{R}$ are considered as structural zeros and their frequencies are trimmed from the maximisation step.

This approach resembles the one adopted by other scholars (see Neycov et al. 2007), in which trimmed estimation is used in a cluster analysis context during the $\mathbf{M}$ step of the EM procedure to avoid inconsistencies in parameters estimation due to the presence of outliers. In the present case, the underlying idea is that $m_{k}$ 's (and $u_{k}$ 's) parameters estimation during the $\mathbf{M}$ step is more robust after trimming those patterns $\gamma$ whose cells, in their respective conditional distributions to the $M$ and $U$ sets, are probably zero under the true model.

In so doing, the relevant part of likelihood (3) with respect to $m_{k}, k=1, \ldots, K$, can be reparametrised as

$$
l\left(g_{\gamma}^{0} N_{\gamma}, \phi\right) \propto \prod_{\gamma \in \Gamma^{S}} \phi_{\gamma}^{\left(g_{\gamma}^{0} N_{\gamma}\right)}
$$

where $\phi_{\gamma}=\frac{m_{\gamma}}{m_{S}}$ are the parameters conditioned to the probability mass $m_{S}=\sum_{\gamma \in \Gamma^{S}} m_{\gamma}$ on
solved patterns $\Gamma^{S}$. Indicating with $M_{S}=\sum_{\gamma \in \Gamma^{S}}\left(g_{\gamma}^{0} N_{\gamma}\right)$ the sum of matched pairs expected in solved patterns and given that $m_{S}+\sum_{\gamma \in \Gamma^{R}} m_{\gamma}=1$ by definition, estimates of $\phi_{\gamma}$ are provided by $\hat{\phi}_{\gamma}=g_{\gamma}^{0} N_{\gamma} / M_{S}, \forall \gamma \in \Gamma^{S}$.

Given the relationship between the sets of parameters $\phi_{\gamma}$ and $m_{\gamma}$ and the independence assumptions (2) that relate $m_{\gamma}$ to their components $m_{k}, k=1, \ldots, K$, it can be written

$$
\frac{\hat{\phi}_{\gamma^{+}}}{\hat{\phi}_{\gamma(-k)}}=\frac{m_{k} \prod_{h \neq k} m_{h}}{\left(1-m_{k}\right) \prod_{h \neq k} m_{h}}=\frac{m_{k}}{\left(1-m_{k}\right)}=\frac{g_{\gamma^{+}}^{0} \cdot N_{\gamma^{+}}}{g_{\gamma(-k)}^{0} \cdot N_{\gamma(-k)}}, \quad k=1, \ldots, K
$$

where:

$-\gamma^{+}=\left\{\gamma_{\mathrm{k}}=1, \mathrm{k}=1, \ldots, \mathrm{K}\right\}$ is the pattern in which all the key variables agree;

$-\gamma(-\mathrm{k})=\left\{\gamma_{\mathrm{k}}=0, \gamma_{\mathrm{i}}=1, \mathrm{i} \in(1, \mathrm{~K}), \forall \mathrm{i} \neq \mathrm{k}\right\}$ are the pattern in which only the $\mathrm{k}$-th key variable disagrees (e.g., for $\mathrm{K}=3, \gamma(-2)=\{1,0,1\}$ ). 
Finally, with some algebraic computations the estimates of $m_{k}, k=1, \ldots, K$ are achieved as

$$
m_{k}^{1}=\frac{g_{\gamma^{+}}^{0} \cdot N_{\gamma^{+}}}{g_{\gamma^{+}}^{0} \cdot N_{\gamma^{+}}+g_{\gamma(-k)}^{0} \cdot N_{\gamma(-k)}}, \quad k=1, \ldots, K,
$$

Similarly, the $u_{k}$ 's are estimated through the multinomial distribution conditioned to the frequencies $\left(1-g_{\gamma}^{0}\right) N_{\gamma}$ on the $\mathrm{K}+1$ patterns $\gamma$ such that $\sum \gamma_{k} \leq 1$ (i.e., patterns in which no more than one key variable agrees) and considering all the other patterns as structural zeros

$$
u_{k}^{1}=1-\frac{\left(1-g_{\gamma^{-}}^{0}\right) N_{\gamma^{-}}}{\left(1-g_{\gamma^{-}}^{0}\right) N_{\gamma^{-}}+\left(1-g_{\gamma(k)}^{0}\right) N_{\gamma(k)}}, \quad k=1, \ldots, K
$$

where

$-\gamma^{-}=\left\{\gamma_{\mathrm{k}}=0, \mathrm{k}=1, \ldots, \mathrm{K}\right\}$ is the pattern in which all key variables disagree;

$-\gamma(\mathrm{k})=\left\{\gamma_{\mathrm{k}}=1, \gamma_{\mathrm{i}}=0, \mathrm{i} \in(1, \mathrm{~K}), \forall \mathrm{i} \neq \mathrm{k}\right\}$ are the patterns in which only the $\mathrm{k}$-th key variable agrees (e.g., for $\mathrm{K}=3, \gamma(2)=\{0,1,0\}$ ).

Again, the procedure is iterated until convergence is reached.

The probabilities $g_{\gamma}=P((a, b) \in M \mid \gamma)$ computed during the E step of EM procedure play another important role in the strategy of assigning pairs to the sets $U$ and $M$. Larsen and Rubin (2001) showed that $g_{\gamma}$ is a monotonic transformation of $r_{\gamma}$ and therefore, inducing the same order on the pairs, these can be used interchangeably. Moreover, $g_{\gamma}$ has a more immediate interpretation and can also be used to estimate the number of pairs incorrectly assigned either to $M$ or $U$ sets. In fact, summing up the corresponding $g_{\gamma}$ for all the pairs assigned to $U$ it is possible to obtain the expected number of missed matches. Conversely, by adding the corresponding $1-g_{\gamma}$ for all the pairs assigned to $M$, the expected number of false matches included among linked units is obtained. These concepts have been taken into account as they will be used in Sections 4 and 5 to assess the proposed procedure.

It is worth noting that robust estimation approach can be applied regardless of the sampling procedure shown in Section 2. In fact, it may be also used when 'lighter' filtering is preferred in order to keep the risk of exclusion of true matches from analysis as low as possible but the ratio between matches and non-matches does not make it possible for the standard estimation method to work.

In the following section, the behaviour of the present approach will be investigated in a simulation context so as to prove its ability in attaining accurate estimates when the match rate falls even far below $0.1 \%$, limit under which the traditional approach is known as not available.

\section{Simulation Study}

As show above, the main limit of classic filtering is that it does not allow the estimation of the probability of matching for all possible pairs from Cartesian product between the files to be matched. Consequently, it is not possible to guess the number of unmatched pairs that could be caused by filtering errors. Although the present method allows overcoming this limit, it is up to us to show that the accuracy of the estimates obtained is adequate. 
As a matter of fact, when considering the present proposal, the accuracy of the estimates can be affected by the following conditions:

1. The sample size of $\Omega^{*}$ from $\Omega$, since the estimates $\hat{p}_{\gamma}$ of the distribution $p_{\gamma}, \forall \gamma \in \Gamma$, are affected by sampling error,

2. The number $\mathrm{K}$ of key variables, because the possible patterns increases as a function of $2^{K}$, while robust estimation approach uses only $2 \cdot(K+1)$ of them, that is, those patterns with either the highest agreement or disagreement between the key variables respectively for $\mathrm{M}$ and $\mathrm{U}$ sets,

3. The magnitude of $u_{k}, k=1, \ldots, K$ probabilities, since even a small increase of $u_{k}$ can raise the number of unmatched pairs that agree on many key variables, when applied to the big amount of pairs $(a, b) \in U$.

This section investigates the accuracy of estimates with respect to the previous points by carrying out a simulation. In order to test the accuracy, various true contingency tables for pairs $(a, b) \in \Omega$ according to patterns of agreement $\gamma \in \Gamma$ between the $\mathrm{K}$ key variables are generated by specifying the following parameters:

- size $N_{A}$ and $N_{B}$ of the files $\mathrm{A}$ and B to be linked,

- size $N_{M}$ of the match set $M$,

- number $K$ of key variables,

- sampling rate $f$ of pairs selected from $\Omega$,

- number of false matches $\varepsilon_{0}$, defined in this analysis as the number of pairs $(a, b) \in U$ whose pattern of agreement is $\gamma \in \Gamma^{S}$, and

- number of missed matches $\varepsilon_{1}$, defined in this analysis as the number of pairs $(a, b) \in M$ whose pattern of agreement is $\gamma \notin \Gamma^{S}$.

For given values of $K, \varepsilon_{0}$ and $\varepsilon_{1}$, the $m_{k}$ and $u_{k}, k=1, \ldots, K$, probabilities are obtained from equations determining the sum of expected frequencies in patterns $\gamma \in \Gamma^{S}$ for nonmatched and matched pairs, respectively,

$$
\begin{gathered}
\left(N_{A} N_{B}-N_{M}\right) P\left(\gamma \in \Gamma^{S} \mid U\right)=\left(N^{2}-N_{M}\right)\left(u^{K}+k \cdot u^{K-1}(1-u)\right)=\varepsilon_{0} \\
N_{M}\left(1-P\left(\gamma \in \Gamma^{S} \mid M\right)\right)=N_{M}\left(1-\left(m^{K}+k \cdot m^{K-1}(1-m)\right)\right)=\varepsilon_{1}
\end{gathered}
$$

where conditional independence (3) is assumed and further restrictions $u_{k}=u$ and $m_{k}=$ $m$, for $k=1, \ldots, K$, are adopted for simplicity.

Once the true frequency table $N_{\gamma}, \forall \gamma \in \Gamma$ is obtained through parameters $n, m$ and $p=\frac{N_{M}}{N_{A} N_{B}}$, the observed frequencies for solved patterns $N_{\gamma}, \forall \gamma \in \Gamma^{S}$ follow directly as a subtable. Sampling frequencies for residual patterns $n_{\gamma}, \gamma \in \Gamma^{\mathrm{R}}$ are instead obtained by sampling from the conditional multinomial distribution with parameters $p_{\gamma}=N_{\gamma} / N_{\Gamma^{\mathrm{R}}}, \forall \gamma \in \Gamma^{\mathrm{R}}$ with sampling ratio $f$ and estimates of $N_{\gamma}, \forall \gamma \in \Gamma^{\mathrm{R}}$ finally resulting from

$$
\hat{N}_{\gamma}=\frac{n_{\gamma}\left(N_{A} N_{B}-\sum_{\gamma \in \Gamma^{S}} N_{\gamma}\right)}{\sum_{\gamma \in \Gamma^{R}} n_{\gamma}}, \forall \gamma \in \Gamma^{\mathrm{R}} .
$$


In the next simulation, 400 sampling tables $n_{\gamma}, \gamma \in \Gamma^{\mathrm{R}}$ were generated by Monte Carlo trials for every set of parameter combinations. A file size of one million records each were considered for files A and B, assuming a perfect match between them, that results in a set $\mathrm{M}$ of one million matches, a set $\mathrm{U}$ of 999.999 billion of non-matches and a marginal matching probability $\mathrm{p}=1 \mathrm{e}-6$, far below the limit that undermines the standard estimation process. These three parameters were kept fixed during the analysis for the sake of brevity, as they did not show any influence on accuracy during non-systematic checks carried out in advance on the data. For every sample, the robust EM algorithm was applied in order to estimate linkage parameters $m_{k}, u_{k}, k=1, \ldots, K$ and $p$ and estimate of $\hat{\varepsilon}_{0}$ and $\hat{\varepsilon}_{1}$ are obtained through Equations (6) and (7). Starting values for EM procedure were kept fixed to $m_{k}=0.9, u_{k}=0.1 k=1, \ldots, K$ and $p=0.01$ after having verified that they do not affect the convergence of the likelihood to its global optimum.

The first evaluation concerned the influence of sampling rate on the accuracy of estimated amounts of false and missed matches. Five sampling rates $f$ were tested, $(0.3$, $0.6,1.0,1.4,1.7$ per million), resulting in samples varying from 300,000 to 1.7 million of pairs.

Figures $1 \mathrm{a}$ and $1 \mathrm{~b}$ below report the sampling distribution for the estimate $\hat{\varepsilon}_{1}$ of missed matches according to increasing sampling rates, for five and nine key variables, when $u$ is fixed so as to provide $\varepsilon_{0}=5,000$ false matches by Equation (6). Both for the present and the following diagrams, the solid line represents the true value, the dashed line describes the sampling median, the dot-dashed line indicates the sampling mean and dotted lines identify respectively $5 \%$ and $95 \%$ quantiles of the sampling distribution. It is possible to note that the distribution of missed matches $\hat{\varepsilon}_{1}$ tends to be less variable and fairly symmetrical for $\mathrm{K}=5$. The distribution is more skewed for $K=9$, and its main part is placed slightly under the true value, since the median and the $5 \%$ quantile both lie under the true value and are very close one another. However, some estimates can be upward biased, as proven by the average value overlaying the true one, while the $95 \%$ quantile is much larger.

Figures $2 \mathrm{a}$ and $2 \mathrm{~b}$ show the sampling distribution of false matches estimates $\hat{\varepsilon}_{0}$ according to increasing sampling rates, for five and nine key variables, when $m$ is fixed so as to provide $\varepsilon_{1}=10,000$ missed matches by Equation (7). Here, it can be noted that

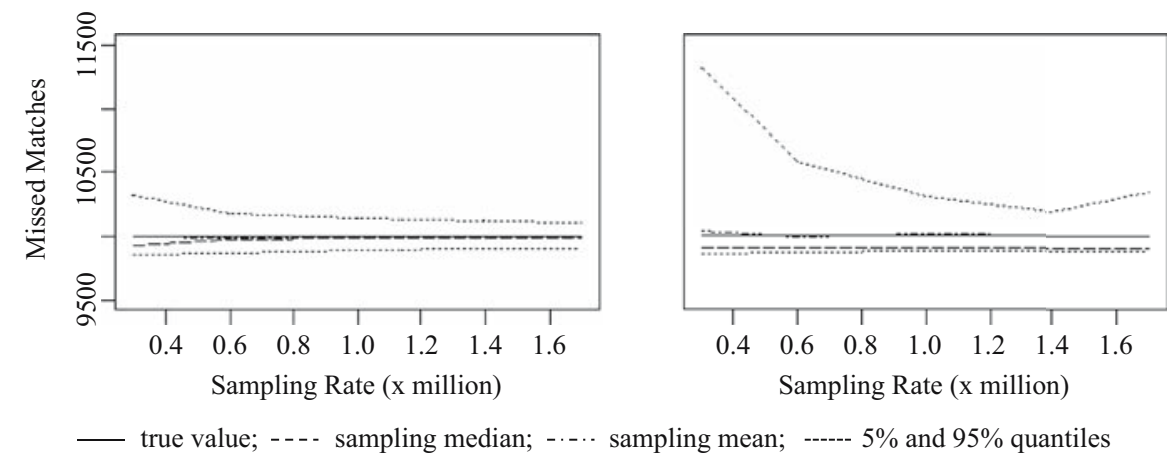

Fig. 1. a. Estimate of 10,000 missed matches by sampling rate - five key variables. b. Estimate of 10,000 missed matches by sampling rate - nine key variables 


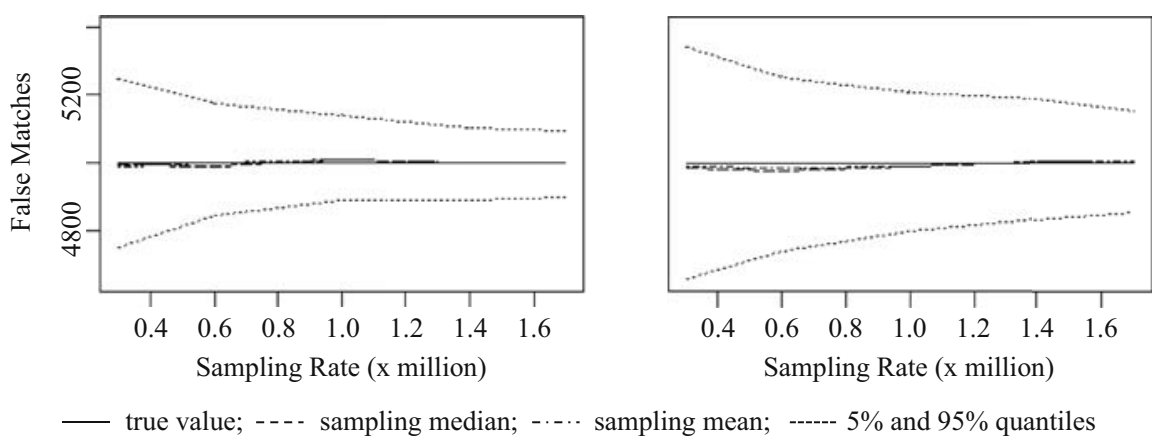

Fig. 2. a. Estimate of 5,000 false matches by sampling rate - five key variables. b. Estimate of 5,000 false matches by sampling rate - nine key variables.

distribution of $\hat{\varepsilon}_{0}$, being mostly of little variability and quite symmetrical for practical purposes, shows less variability for $\mathrm{K}=5$ and for large sampling rates.

Figure 3 summarises the sample distribution of the estimated missed matches amount $\hat{\varepsilon}_{1}$ according to increasing number of false matches, in case of five key variables. A sample of one million pairs was considered for these tests. Surprisingly enough, an anomalous behaviour of the sampling distribution is observed for missed matches estimates when the incidence of false matches is very small. The median of the distribution is very close to the $5 \%$ quantile and both are very close to the true value.

The $95 \%$ quantile is instead much larger than the true value, denoting the risk of outliers in the estimate of $\varepsilon_{1}$ when data are affected by only few false matches (i.e., probabilities $u_{k}, k=1, \ldots, K$ are very small). When the number of false matches increases to 50,000 pairs, the sampling distribution of missed matches becomes symmetrical while its variability decreases, until the number of false matches becomes larger and variability starts to increase significantly again. It is worth noting that, even in the worst-case scenario, estimates are not further from the true value than about $10 \%$.

In Figure 4 , the distribution of missed matches $\hat{\varepsilon}_{1}$ as a function of false matches is reported for $K=9$. The distribution performs better than the corresponding one for $K=5$, since it is almost perfectly close to the real value when false matches are few, with negligible variability and a relative bias of less than $1 \%$. The variability of the distribution

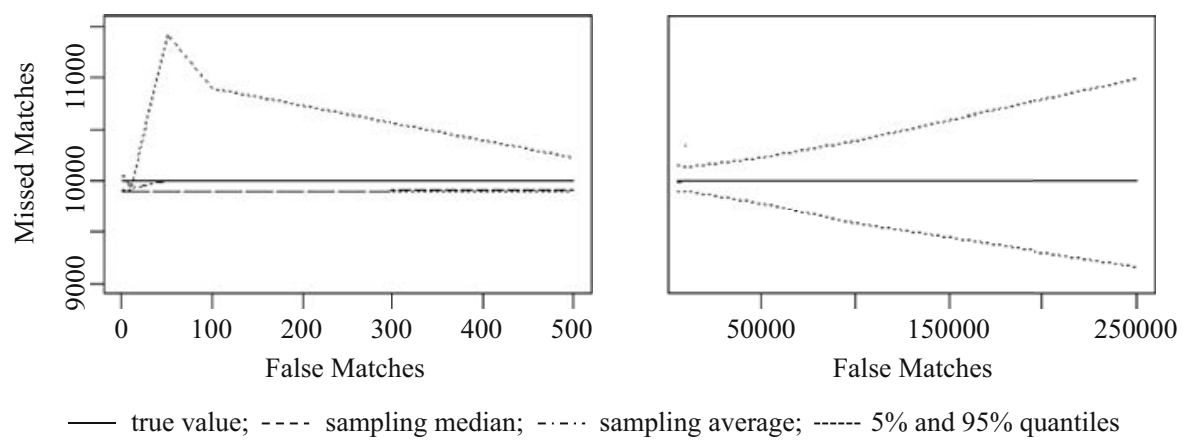

Fig. 3. Estimate of 10,000 missed matches by false matches - five key variables. 


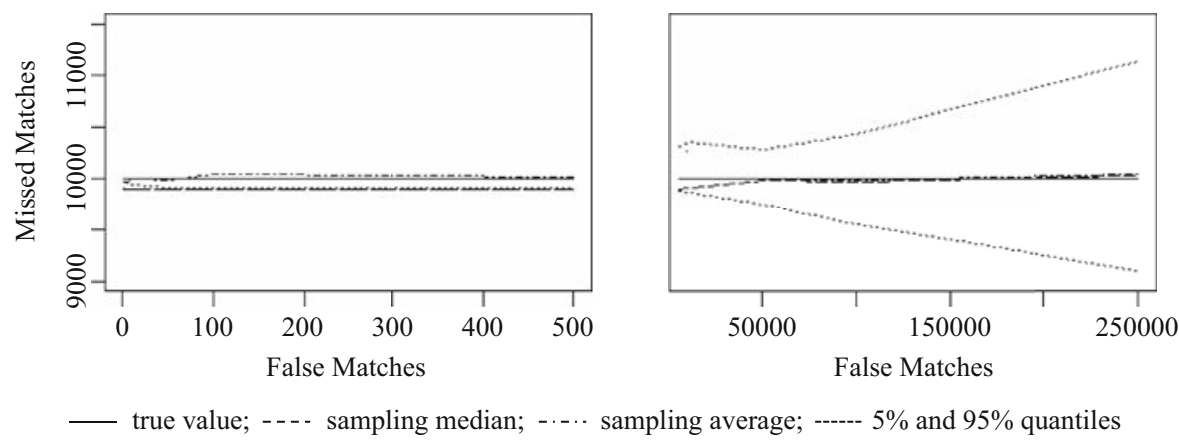

Fig. 4. Estimate of 10,000 missed matches by false matches - nine key variables.

continues to be small until the missed matches do not exceed 50,000, a quite pessimistic scenario corresponding to $5 \%$ of the total number of matches considered in the current simulation. This behaviour corroborates the accuracy of estimates when the number of key variables increases and at least one million pairs are sampled from the $10^{12}$ pairs in $\Omega$.

Finally, Figures 5 and 6 show the sampling distribution of the estimated number of false matches $\hat{\varepsilon}_{0}$ by increasing the number of missed matches, for five and nine key variables,

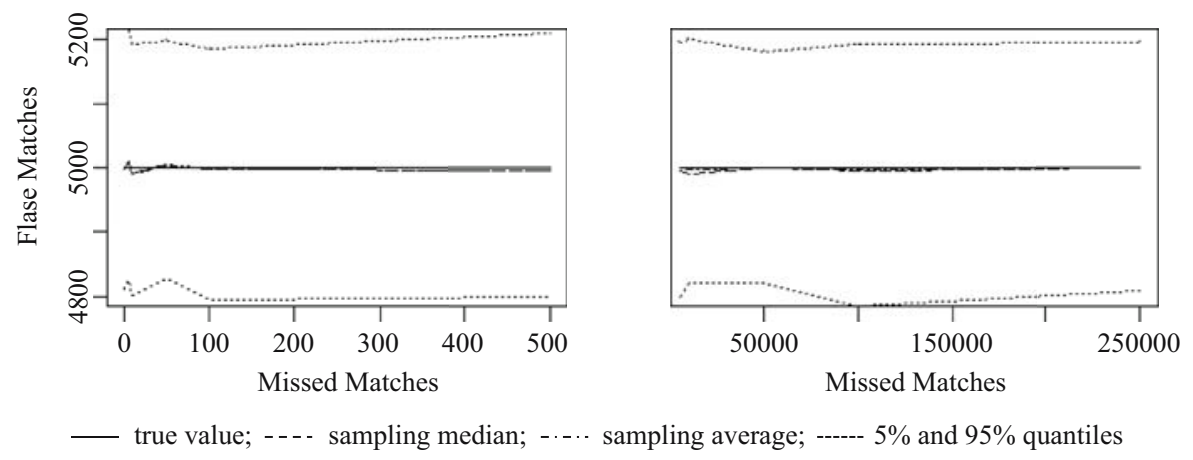

Fig. 5. Estimate of 5,000 false matches by missed matches - five key variables.
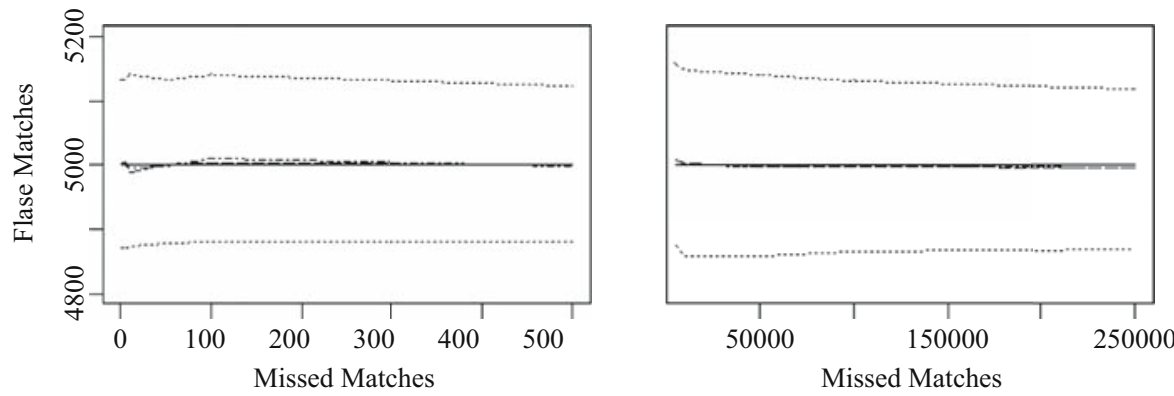

_ true value; -.-- sampling median; -... sampling average; ----- 5\% and $95 \%$ quantiles

Fig. 6. Estimate of 5,000 false matches by missed matches - nine key variables. 
respectively. It can be noted that the estimates are unbiased, have a symmetric distribution and are not affected by the incidence of missed matches, while the number of the key variables influences only marginally its variability.

\section{A Real Case Study}

This section provides an application of the method to real data and makes a comparison with a common filtering approach. The goal is to show how the linkage strategy presented above works when applied to a common case where (at least) one of the key variables is compared through a fuzzy string similarity distance (Herzog et al. 2007). Furthermore, a focus will be put on how the estimate of linkage probability for all the possible pairs can be used in practice for identifying further matches in patterns previously excluded by the filtering step.

The aim is not to make an exhaustive comparison between the present method and the most advanced filtering techniques. Such a comparison is beyond the scope of the present work since, to our knowledge, no advanced filtering method can return the linkage probability for pairs excluded from analysis, which our approach is able to do. For this reason, we choose to compare our approach with a sorted neighbour filtering (Hernandez and Stolfo 1995) which allows to appreciate the differences between approaches without unnecessarily complicating the exposition.

In our example, a file of 16,723 foreigners who applied for a permit to stay (PS) is linked to another file of 19,398 foreigners registered in the municipal population registry lists (MPR). Although the size of the two files is not excessively large, in this case the Cartesian product between them results in more than 324 million of possible pairs and the standard Fellegi-Sunter approach already needs of a filtering step in order to be applied without bias. Since, by definition, no more than one record of the larger file can be matched to each record of the smaller one, the proportion $p$ of matches over all the possible pairs cannot exceed the rate of about $5 \cdot 10^{-5}$, in this case $(1 / 19,398)$. The example is taken from a preparatory application to the 2011 Italian census, which aimed to fetch a contact list of foreigners not already registered in population registers among the appliers for a "permit to stay" (Fortini et al. 2013). The linkage meant to identify people in the PS file who were not already included in the population register, so as to be contacted during the census. In our example, data concerning the Abruzzo region are investigated in order to identify people enlisted in both sources.

The key variables used to identify individuals in both the lists are: First and last name (single field), Gender, Country of citizenship code, Day of birth, Month of birth and Year of birth.

Although robust estimation assisted by sampling filtering is, in our opinion, intrinsically better than classic approach as virtually including all possible pairs in the evaluation of their probability of linkage, the results of the proposed application will be briefly compared with those obtained by filtering with 'sorted neighbours' algorithm on the variable 'First and last name'.

As far as the quality of input data is concerned, the amount of missing values affecting key variables can be considered negligible, since the MPR source is affected by missing data only on the variable 'First and last name' at a $0.01 \%$ level, while PS shows $5 \%$ of 
missing data on 'Gender' and an abnormal concentration of births occurring on the first day of January (about 750 cases against average values between 50 and 70 for the other dates of the year), which could affect the discriminatory power of the day and month of birth.

The pairwise comparison between key variables is carried out by means of dichotomous indicators that assumes value 1 in case of agreement and 0 otherwise. The agreement is established when both the instances of the key variable have the same value, except for missing values whose presence always gives rise to a disagreement. Only for the variable 'First and last names', the comparison was grounded on the three-gram Jaccard string distance, which always ranges between 0 and 1 (Christen 2012), and is labelled as agreement when exceeding 0.7 and as disagreement otherwise.

In order to achieve the frequency distribution of pairs by solved patterns, the procedure presented in Section 2 was applied to all the key variables with exclusion of 'First and last names'. In fact, the agreement/disagreement label to 'First and last names' was assigned after the evaluation of the Jaccard distance to all the pairs falling within the patterns recognised by the other key variables. With regard to the effort for completing this phase of data processing, 60,616 pairs were checked.

A random sample of 500,000 pairs was subsequently selected from the Cartesian product of the two files to be linked by means of two independent random selections with replacement of 500,000 records from the files and their consequent inclusion, which finally reduces to 499,642 pairs after the exclusion of duplicates. Table 3 shows the frequency distribution of pairs according to solved (bolded rows) and remaining patterns, whose frequencies are estimated through the sample. It can be noted that sampling zeros are achieved for some of the patterns characterized by high concordance between key variables, since their expected frequencies are fairly low. Since these patterns are not directly used for estimation of parameters $m_{k}$ and $u_{k}$, these flaws do not invalidate the method. Nevertheless, they can affect the precision of the expected number of matches for these specific patterns and offer a prospect for future improvement on sample design and model estimation of observed frequencies for remaining patterns.

Data in Table 3 was used for estimation of linkage parameters with robust EM, as described in Section 3. Starting values $\mathrm{m}_{\mathrm{k}}=0.9, \mathrm{u}_{\mathrm{k}}=0.1, \mathrm{k}=1, \ldots, 6$, were assigned to each of the six key variables, while starting value $p$ was fixed at 0.01 . Tests carried out with many different starting values does not change the estimates resulting from the method, but only affects the number of iterations needed for the algorithm to converge. Under these conditions, the robust EM algorithm converges in about 50 iterations, returning $\mathrm{p}=1.86 \mathrm{E}-5$ and the $m$ 's and $u$ 's values shown in the Table 4 .

From rates $r_{k}$ between $m_{k}$ and $u_{k}$, for $k=1, \ldots, 6$, it can be seen that 'First and last names' has the highest discriminant power followed by 'Day of birth', 'Year of birth', 'Country ID', 'Month of birth' and finally 'Gender'. Expected frequencies of matched and non-matched pairs by their patterns of agreement (solved patterns are reported in bold) are shown in Table 5, as well as the matching probabilities conditional to the pattern. The patterns are sorted by descending probabilities $P(M \mid \gamma)$ so as to identify those having a higher number of true matches with minimum inclusion of false ones.

By only considering the solved patterns and adopting a threshold of 0.5 on $P(M \mid \gamma)$, the number of matches expected to be retrieved is 5,172 plus one false match and 26 missed 


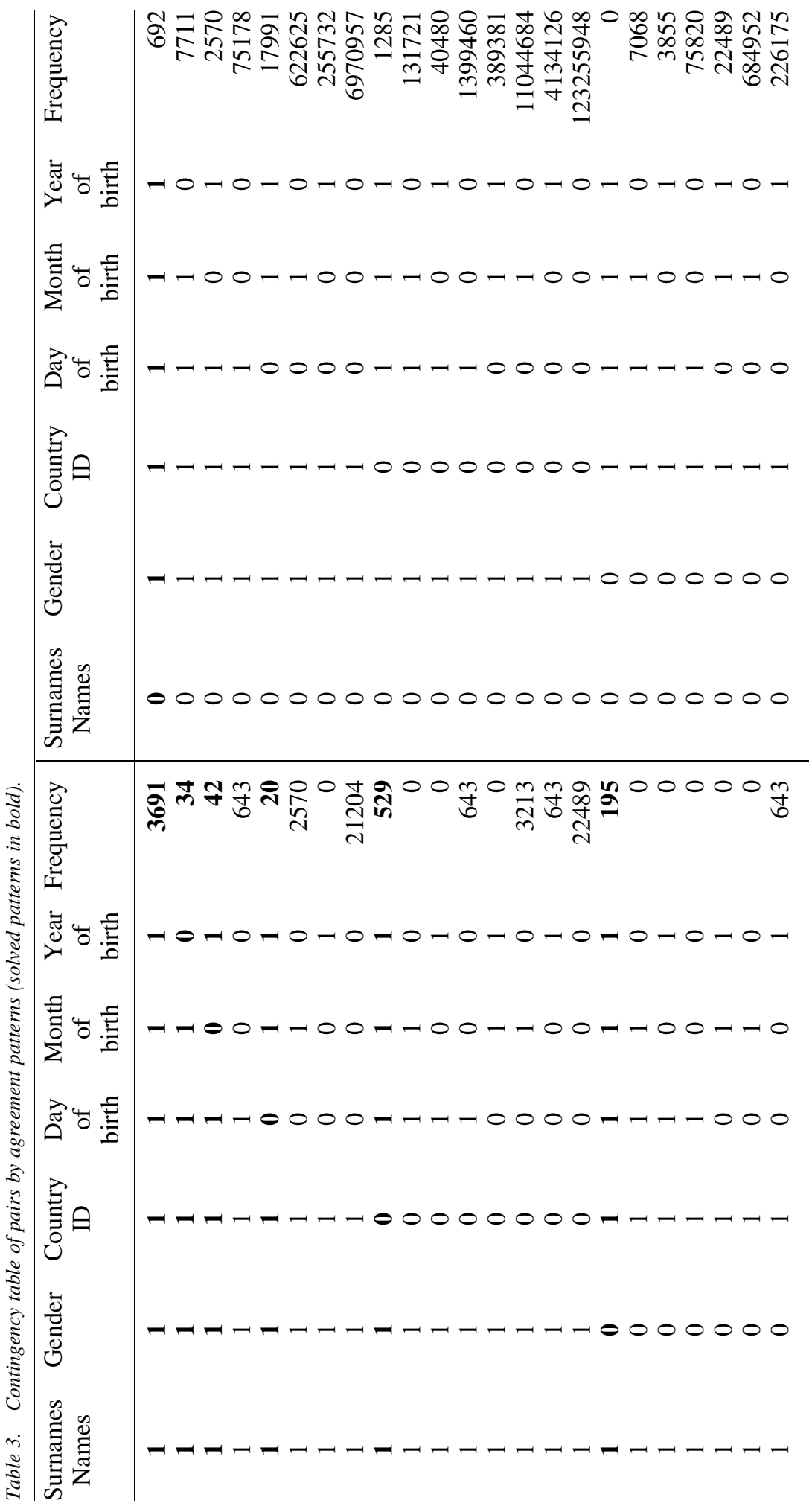




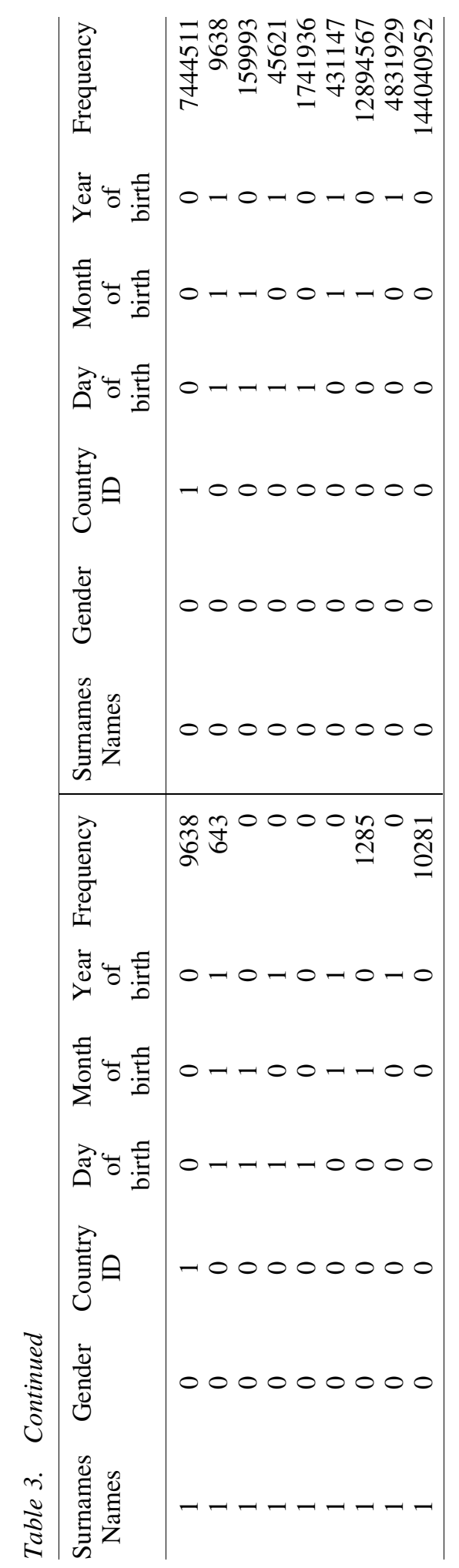


Table 4. Marginal probability of agreement for each key variable depending on the match status of the pair.

\begin{tabular}{lllllll}
\hline & $\begin{array}{c}\text { First and } \\
\text { last names }\end{array}$ & Gender & $\begin{array}{c}\text { Country } \\
\text { id }\end{array}$ & $\begin{array}{c}\text { Day } \\
\text { of birth }\end{array}$ & $\begin{array}{c}\text { Month } \\
\text { of birth }\end{array}$ & $\begin{array}{c}\text { Year } \\
\text { of birth }\end{array}$ \\
\hline$k$ & 1 & 2 & 3 & 4 & 5 & 6 \\
$m_{k}$ & 0.984 & 0.887 & 0.790 & 0.957 & 0.964 & 0.963 \\
$u_{k}$ & 0.000 & 0.461 & 0.049 & 0.012 & 0.082 & 0.032 \\
\hline
\end{tabular}

ones. In real cases, a certain amount of clerical work is spent to scrutinise pairs whose probability $P(M \mid \gamma)$ is lower than a fixed value so as to distinguish other true matches, which is not done here for practical reasons. However, if the whole table is considered, the number of expected matches increases to 6,037, with a difference that leads to examine other patterns that have high match probability $P(M \mid \gamma)$. Table 5 shows that 729 additional matches are expected from the scrutiny of nine residual patterns in which $P(M \mid \gamma) \geq 0.5$, together with a concomitant inclusion of 54 more false match pairs. Nonetheless, sampling variability affects this figures due to sample survey on the set of remaining patterns and, subsequent to investigation of nine patterns, the number of additional true matches is reduced to 273 , with an inclusion of 29 more false matches.

This additional work requires the screening of 811,341 pairs which, added to the 60,616 and 500,000 pairs already considered, brings to $1,371,957$ the total number of pairs taken into account, out of the more than 324 million of couples from the Cartesian product across the files. Consequently, 5,475 links are found overall, 30 of which are expected to be false, while 592 matches are expected to be missed across patterns with $P(M \mid \gamma)<0.5$.

In order to compare the example above with the standard approach, the linkage exercise was repeated after filtering the set of pairs by a 'Sorted neighbours' procedure on the variable 'First and last name' with a comparison window of 50 units. Among other things, the attempt to expand the window to 100 units produces about 1.7 million pairs to analyse and leads to the failure of the standard estimation procedures of the probability of linkage. The adopted filtering selection sets the pairs to 861,528, on which the standard EM estimation approach works furtherly by returning a marginal probability $p=0.006$. This probability is 200 times larger than the one estimated by the present method, supporting the fact that filtering increased the ratio between matches and non-matches among pairs retained in analysis. Vectors of parameters $m_{k}$ and $u_{k}$, estimated through the standard method, are shown in Table 6 to be compared with those previously reported in Table 4 . It can be noted that, despite a fairly similar overall values for $m_{k}$ and $u_{k}$, the probabilities $u_{k}$ related to non-match pairs increases for 'Country ID' and 'First and last Names', with a change in their rank in terms of discriminatory power compared with other key variables.

On considering patterns that have posterior probabilities $\mathrm{P}(M \mid \gamma) \geq 0.5$, the filtered approach gives rise to 5,129 links, 5,102 expected true match and 18 false, with 27 missing matches remaining among patterns with $\mathrm{P}(M \mid \gamma)<0.5$. In comparison, the present approach has achieved better results even taking into account only the solved patterns (with 5,172 true match and only one false match). In addition, it is worth noting that filtering method probably excluded more than 900 matches from the analysis, as highlighted by the difference between the 6,037 , expected by our method, and the 5,129 estimated by the sorted neighbour example. This difference would not have been detected if we had not used the proposed approach. 


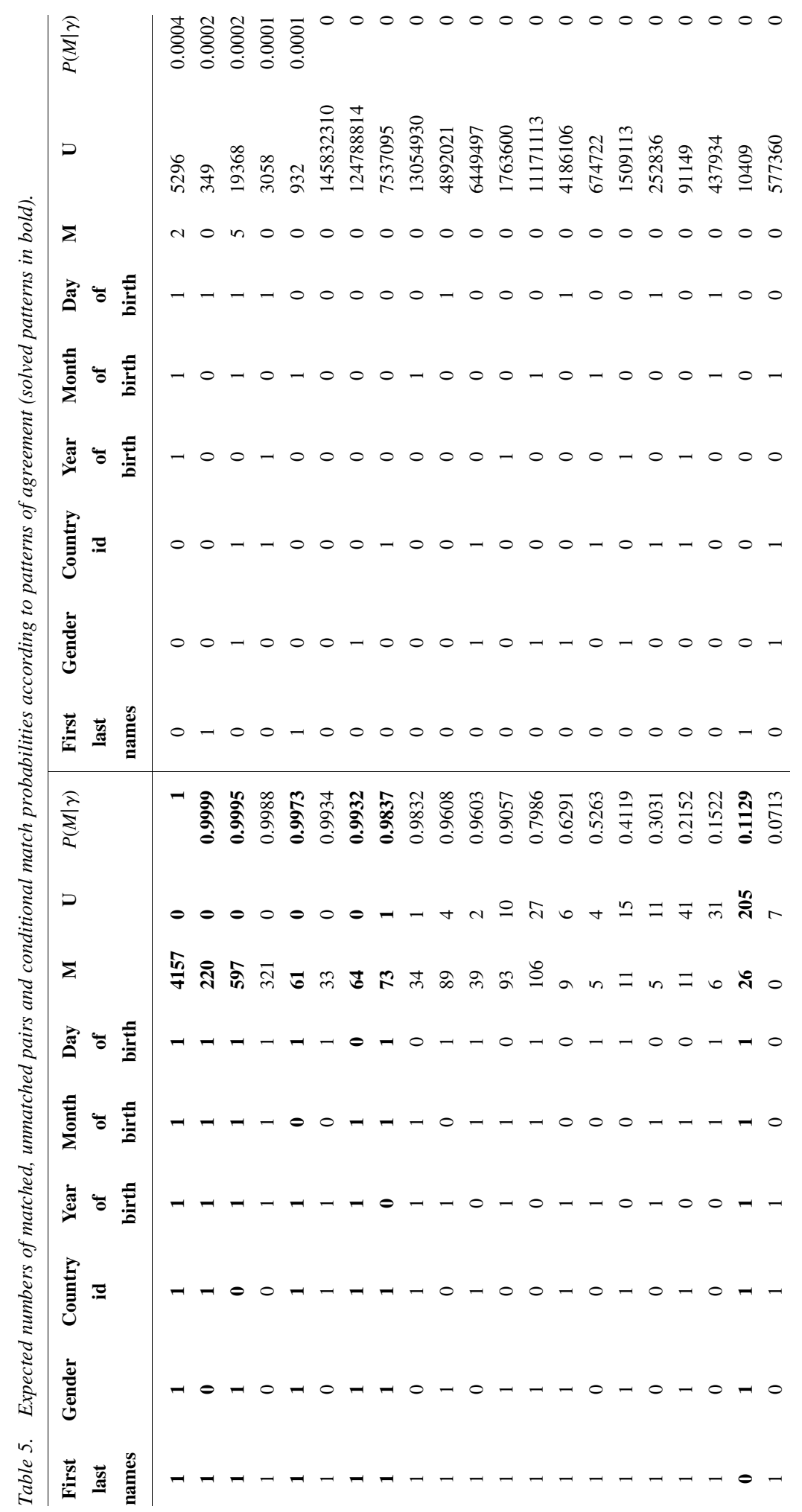




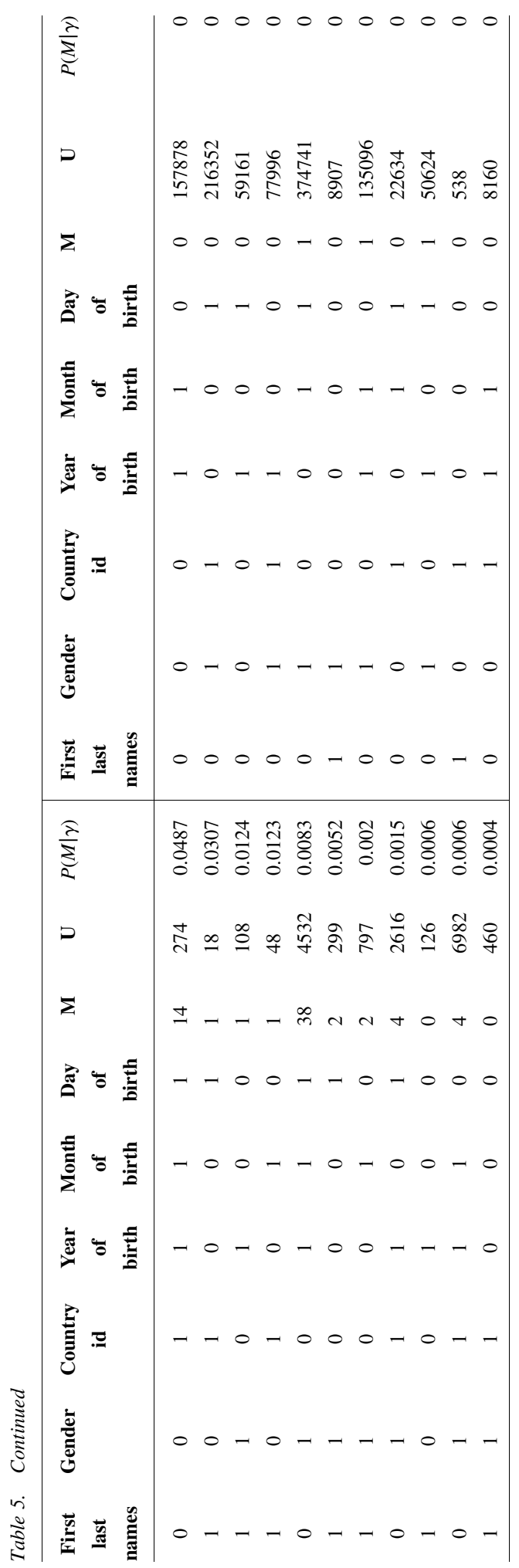


Table 6. Sorted Neighbours: marginal probability of agreement for each key variable conditional to match status of the pair.

\begin{tabular}{llllccc}
\hline & $\begin{array}{c}\text { First and } \\
\text { last names }\end{array}$ & Gender & $\begin{array}{c}\text { Country } \\
\text { id }\end{array}$ & $\begin{array}{c}\text { Day } \\
\text { of birth }\end{array}$ & $\begin{array}{c}\text { Month } \\
\text { of birth }\end{array}$ & $\begin{array}{c}\text { Year } \\
\text { of birth }\end{array}$ \\
\hline$k$ & 1 & 2 & 3 & 4 & 5 & 6 \\
$m_{k}$ & 0.997 & 0.935 & 0.863 & 0.991 & 0.989 & 0.987 \\
$u_{k}$ & 0.026 & 0.479 & 0.201 & 0.012 & 0.082 & 0.032 \\
\hline
\end{tabular}

The improvement of the present method if compared to the standard one becomes even more evident when further effort is carried out to consider the nine additional patterns with posterior probability $\mathrm{P}(M \mid \gamma) \geq 0.5$, thus, achieving a whole number of 5,445 true match and 30 false matches. However, considering that such better results are obtained by checking 510,429 pairs more than those examined for the filtered case, it is possible to limit the present method to probe only additional profiles whose $\mathrm{P}(M \mid \gamma) \geq 0.9$. With this tighter limit, the pairs examined in the two cases become almost the same $(851,628$ against 861,628$)$, while the gain of the present method remains significant. In fact, 5,364 linked pairs are obtained and only five of them are expected to be false, that is, 230 true matches more and 13 false matches less.

\section{Final Remarks}

This article introduces sampling features to probabilistic record linkage and extends the model estimation capabilities to large files while remaining in a Fellegi-Sunter framework. In this way, computational efforts were managed without affecting the set of pairs object of the study. It outperforms traditional filtering methods in that it accomplishes the estimation of linkage probabilities as to the whole set of pairs, which would otherwise be excluded from the analysis. In addition, it is effective in driving further analysis on patterns that have not been solved but that show evidence of including a high number of matches at the end of the estimation phase.

In addition to the simulations demonstrating the accuracy of the method, a real case application was presented to show how it can be used in practice for record linkage. The example is also useful to see how the method works when key variables are compared by fuzzy string similarity functions.

In our opinion, trials on larger datasets, in which filtering techniques are subject to more failures, will take advantage of this method to its fullest extent. Nevertheless, we believe that the robust approach to the estimation of linkage probabilities can be useful 'per se' to reduce filtering as much as possible, in such a way reducing the risk of excluding by mistake true matches from the analysis. On the other hand, our sample based filtering could be used in addition to a given standard filtering method to verify that the latter does not exclude too many "valid" pairs (matches) by mistake before the linkage step.

Further improvements include efficient sampling schemes on the set of pairs $\Omega$ to make better inferences on the contingency table of pairs by their pattern of agreement. Moreover, the use of log-linear models might improve the estimate of small frequencies for sampled patterns having small frequencies (patterns with a relatively high agreement between key variables) so as to mitigate the side effects of sampling zeros.

A better estimate of frequency distribution could also be exploited by the robust EM approach, after it is properly adjusted in order to release some of the conditional 
independence assumptions between key variables. In doing so, a certain degree of association between key variables could be considered in order to improve the estimate of linkage probabilities as suggested by Thibaudeau (1993).

Other developments could be related to specific strategies to adapt the probabilistic procedure to deterministic linkage steps carried out in advance. For example, a scheme that, after a deterministic linkage based on perfect agreement between a given set of key variables, applies a probabilistic step to records not linked at previous stage by using the same set of key variables, could be developed. In this case, a structural zero should be considered for patterns of perfect agreement already used during the deterministic step, and, as a consequence, an appropriate conditional probabilistic model is to be defined.

\section{References}

Bishop, Y.M., S.E. Fienberg, and P.W. Holland. 1975. Discrete multivariate analysis. Cambridge, Mass.: MIT Press. DOI: https://doi.org/10.1007/978-0-387-72806-3.

Baxter, R., P. Christen, and T. Churches. 2003. “A Comparison of Fast Blocking Methods for Record Linkage”. CMIS Technical Report 03/139, six-pages version of the paper published in Proceedings of ACM SIGKDD '03. Available at: https://citeseerx.ist.psu.edu/viewdoc/ download?doi=10.1.1.10.4563\&rep= rep1\&type=pdf (accessed April 2020).

Christen, p. 2012. Data matching: concepts and techniques for record linkage, entity resolution, and duplicate detection. Springer Science and Business Media. DOI: https:// doi.org/10.1007/978-3-642-31164-2.

Cibella, N., M. Fortini, M. Scannapieco, L. Tosco, and T. Tuoto. 2009. "Theory and practice in developing a record linkage software”. Insights on Data Integration Methodologies: 37-56. Available at: https://ec.europa.eu/eurostat/documents/3888793/5845197/KS-RA-09-005EN.PDF/4cef0f2d-45a0-46b7-bfd6-196a55fca801?version=1.0 (accessed April 2020).

Cormen, T.H., C.E. Leiserson, R.L. Rivest, and C. Stein. 2009. Introduction to algorithms. MIT press. Available at: https://mitpress.mit.edu/books/introduction-algorithms-thirdedition (accessed April 2020).

Dempster, A.P., N.M. Laird, and D.B. Rubin. 1977. "Maximum Likelihood from Incomplete Data via the EM Algorithm". Journal of the Royal Statistical Society B 39: 1-38. DOI: https://doi.org/10.1111/j.2517-6161.1977.tb01600.x.

Hernandez, M.A., and S.J. Stolfo. 1995 "The merge/purge problem for large databases". Edited by M.J. Carey and D.A. Schneider in SIGMOD, 127-138. DOI: https://doi.org/ 10.1145/568271.223807.

Herzog, T.N., F.J. Scheuren, and W.E. Winkler. 2007. Data quality and record linkage techniques. Springer Science and Business Media. DOI: https://doi.org/10.1007/0-38769505-2.

Fellegi I., and A.B. Sunter. 1969. "A Theory for Record Linkage", Journal of the American Statistical Association, 64, 328: 1183-1210. DOI: https://doi.org/10.1080/ 01621459.1969.10501049.

Fortini, M., L. Mancini, L.Marcone, E.Mussino, and E. Paluzzi. 2013. "Who Settles Down in Italy? Transition to Residency of non-EU Migrants". Rivista Italiana di Economia Demografia e Statistica, no. LXVII, (3/4). Available at: https://www.sieds.it/listing/ RePEc/journl/2013LXVII_N34rieds.pdf (accessed April 2020). 
Jaro, M.A. 1989. “Advances in Record-Linkage Methodology as Applied to Matching the 1985 Census of Tampa, Florida”. Journal of the American Statistical Association, 84: 414-420. DOI: https://doi.org/10.1080/01621459.1989.10478785.

Larsen, M.D., and D.B. Rubin. 2001. "Iterative automated record linkage using mixture models". Journal of the American Statistical Association, 96(453): 32-41. DOI: https:// doi.org/10.1198/016214501750332956.

Murray, J. 2015. "Probabilistic Record Linkage and Deduplication after Indexing, Blocking, and Filtering". Journal of Privacy and Confidentiality, 7(1). DOI: https://doi. org/10.29012/jpc.v7i1.643.

Neykov, N., P. Filzmoser, R. Dimova, and P. Neytchev. 2007. "Robust fitting of mixtures using the trimmed likelihood estimator". Computational Statistics \& Data Analysis, 52(1): 299-308. DOI: https://doi.org/10.1016/j.csda.2006.12.024.

Newcombe, H.B., J.M. Kennedy, S.J. Axford, and A.P. James. 1959. “Automatic linkage of vital records”. Science, 130(3381): 954-959. DOI: https://doi.org/10.1126/science. 130.3381.954.

Thibaudeau Y. 1993. "The discrimination power of dependency structures in record linkage". Survey Methodology, 19: 31-38. Available at: https://www150.statcan.gc.ca/ n1/pub/12-001-x/1993001/article/14477-eng.pdf (accessed April 2020).

Winkler, W.E. 1988. "Using the EM Algorithm for Weight Computation in the FellegiSunter Model of Record Linkage". Proceedings of the Section on Survey Research Methods: American Statistical Association: 667-671. Available at: https://www. asasrms.org/Proceedings/papers/1988_124.pdf (accessed April 2020).

Winkler, W.E. 1989. "Near Automatic Weight Computation in the Fellegi-Sunter Model of Record Linkage". Proceedings of the Fifth Census Bureau Annual Research Conference, March 19-22, Arlington, Virginia, U.S.A.: 145-155. Available at: https://www.academia.edu/34177520/Near_Automatic_Weight_Computation_in_the_Fellegi-Sunter_Model_of_Record_Linkage (accessed April 2020).

Winkler, W.E. 2006. "Overview of record linkage and current research directions". Bureau of the Census Working Paper No. RRS2006-02. Available at: https://www.census.gov/library/working-papers/2006/adrm/rrs2006-02.html (accessed April 2020).

Yancey, W.E. 2002. "Improving EM Algorithm Estimates for Record Linkage Parameters". Proceedings of the Section on Survey Research Methods: American Statistical Association. Available at https://www.asasrms.org/Proceedings/y2002/Files/ JSM2002-000581.pdf (accessed April 2020).

Received July 2018

Revised May 2019

Accepted July 2020 